\title{
Physical sources of sound in laminar and turbulent jets
}

$\begin{array}{lll}\text { S. Sinayoko A. Agarwal R. D. Sandberg } & \end{array}$

Southampton

Institute of Sound and

Vibration Research

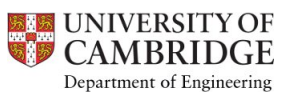

SouthNWIVERSTY of
School of Engineering sciences

$17^{\text {th }}$ AIAA/CEAS Aeroacoustics Conference

Portland - 8 June 2011 


\title{
Introduction
}

\author{
Aim \\ To understand the physical sources of jet noise.
}




\section{Introduction}

\section{Aim \\ To understand the physical sources of jet noise.}

Motivation

- By-pass ratio is limited

- Find alternative strategies 


\section{Introduction}

\section{Aim \\ To understand the physical sources of jet noise.}

\section{Motivation}

- By-pass ratio is limited

- Find alternative strategies

\section{Methods}

- Non-radiating base flow sources

- DNS 


\section{Introduction}

\section{Aim}

To understand the physical sources of jet noise.

\section{Motivation}

- By-pass ratio is limited

- Find alternative strategies

\section{Previous work}

Single frequency algorithm

\section{Methods}

- Non-radiating base flow sources

- DNS 


\section{Introduction}

\section{Aim}

To understand the physical sources of jet noise.

Motivation

- By-pass ratio is limited

- Find alternative strategies

Methods

- Non-radiating base flow sources

- DNS
Previous work

Single frequency algorithm

Objectives of the presentation

- Multiple frequency algorithm

- Sources in a laminar jet

- Sources in a turbulent jet 


\section{Part I}

Non-Radiating Base Flow (NRBF) Sources 


\section{NRBF sources definition}

$$
f_{i}=-\frac{\partial}{\partial x_{j}}\left(\bar{\rho} \widetilde{v_{i}} \widetilde{v}_{j}\right)^{\prime}
$$

$$
\widetilde{v_{i}}=\overline{\rho v_{i}} / \bar{\rho}
$$




\section{Flow decomposition}

Space-time domain

$$
q^{\prime}=w * q
$$

$$
q(\mathbf{x}, t) \stackrel{\text { F.T. }}{\longrightarrow} Q(\mathbf{k}, \omega)
$$

$q^{\prime}(\mathbf{x}, t)+\left\{\begin{array}{ll|}1 & \text { if }|\mathbf{k}|=|\omega| / c_{\infty} \\ 0 & \text { otherwise }\end{array}\right.$ 


\section{Part II}

\section{Sound sources in a laminar jet}




\section{Flow description}

Pressure field

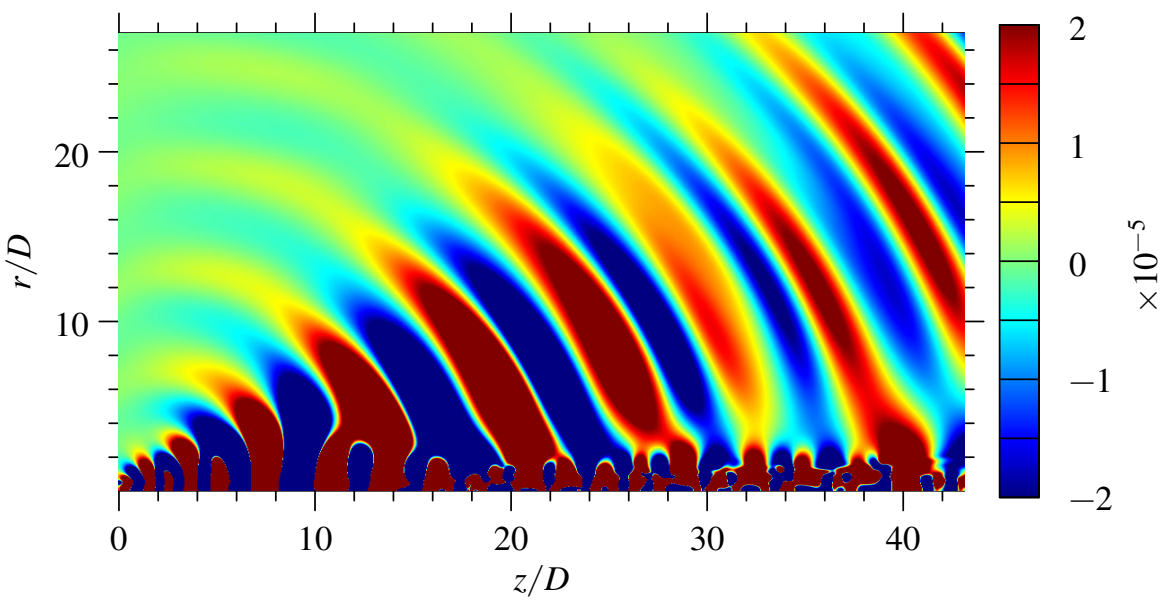



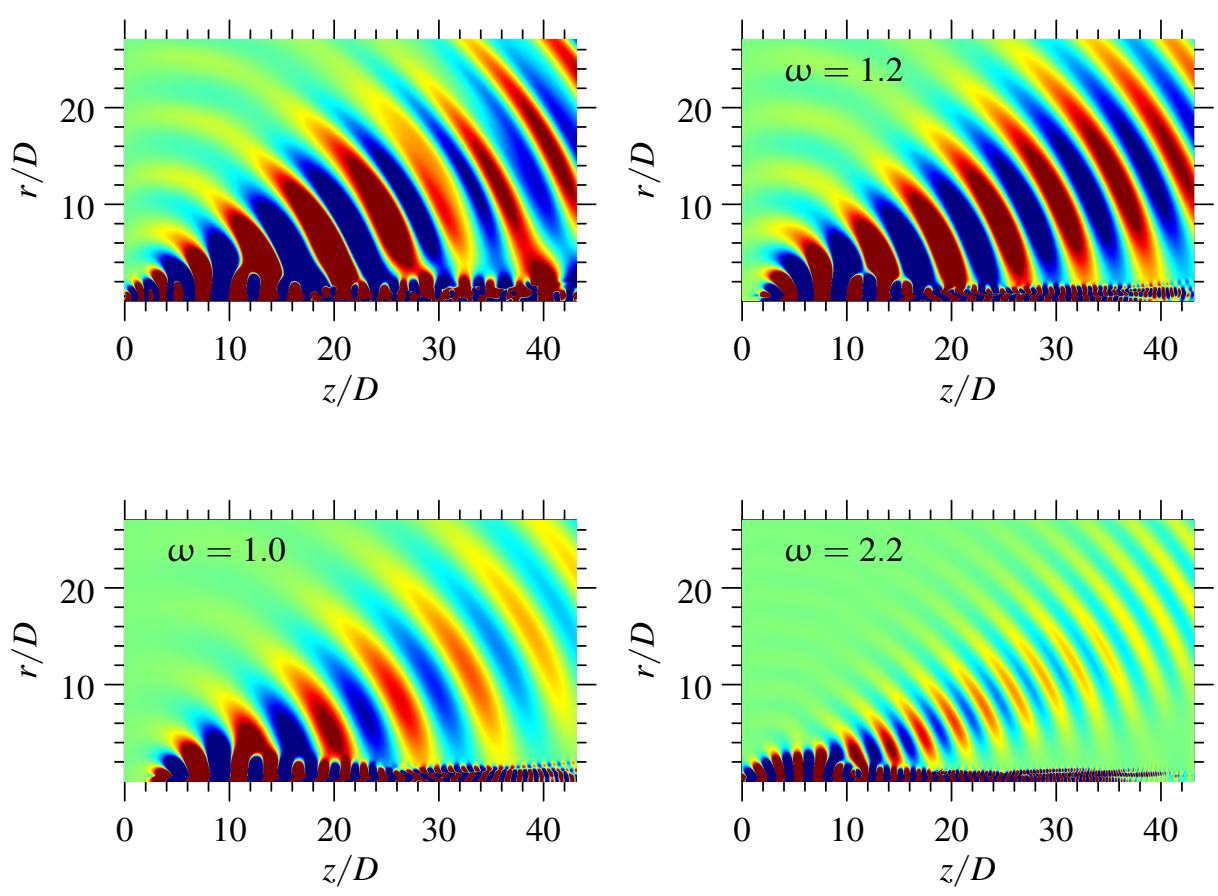


\section{Pressure in Fourier domain $(\omega=1.2)$}

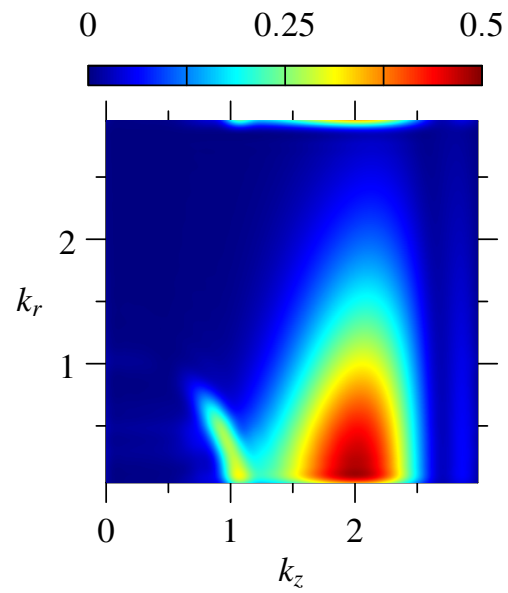

Pressure $P_{1.2}(\mathbf{k})$ 
Pressure in Fourier domain $(\omega=1.2)$

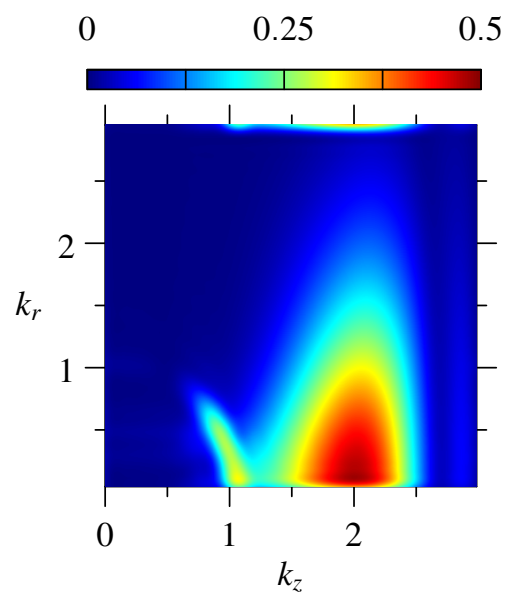

Pressure $P_{1.2}(\mathbf{k})$

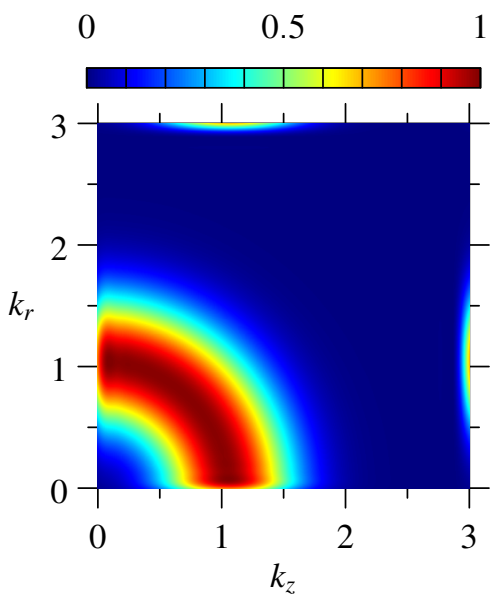

Gaussian filter $W(\mathbf{k})$ 


\section{Pressure in Fourier domain $(\omega=1.2)$}

\section{Decomposition}

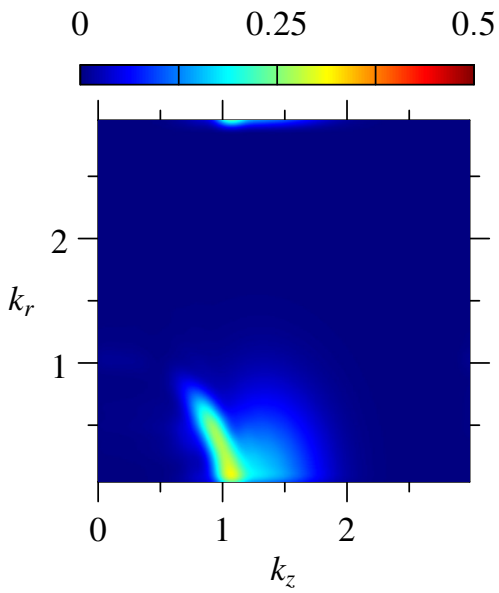

Radiating pressure

$$
P_{1.2}^{\prime}=W P
$$




\section{Pressure in Fourier domain $(\omega=1.2)$}

\section{Decomposition}

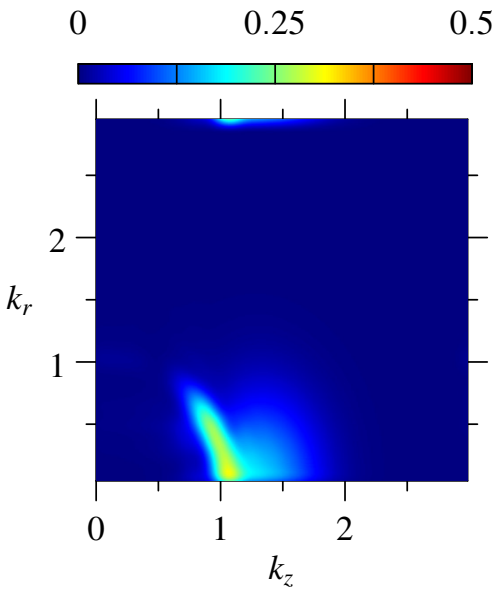

Radiating pressure

$$
P_{1.2}^{\prime}=W P
$$

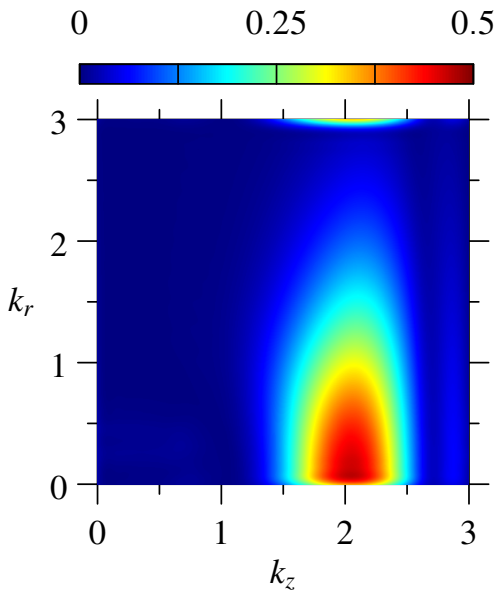

Non-radiating pressure

$$
\bar{P}_{1.2}=(1-W) P_{1.2}
$$




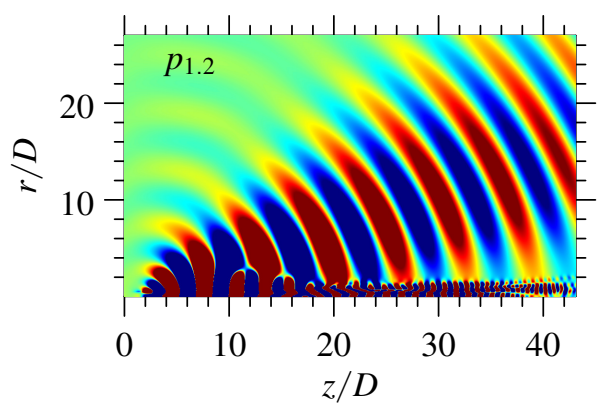



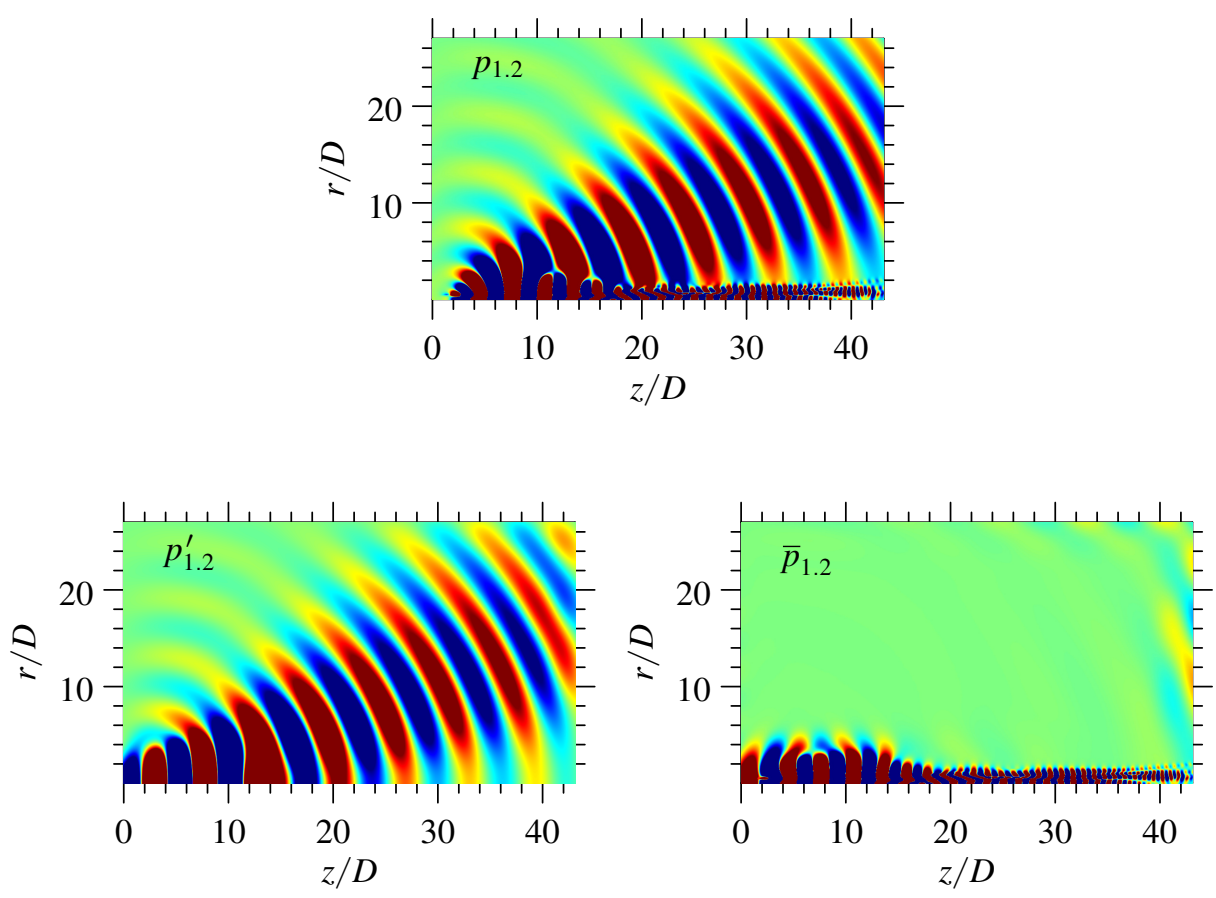


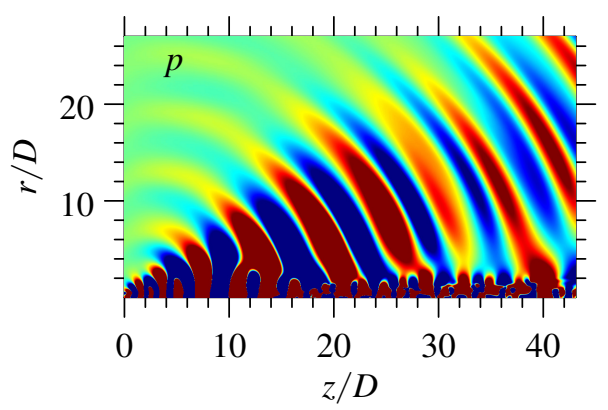



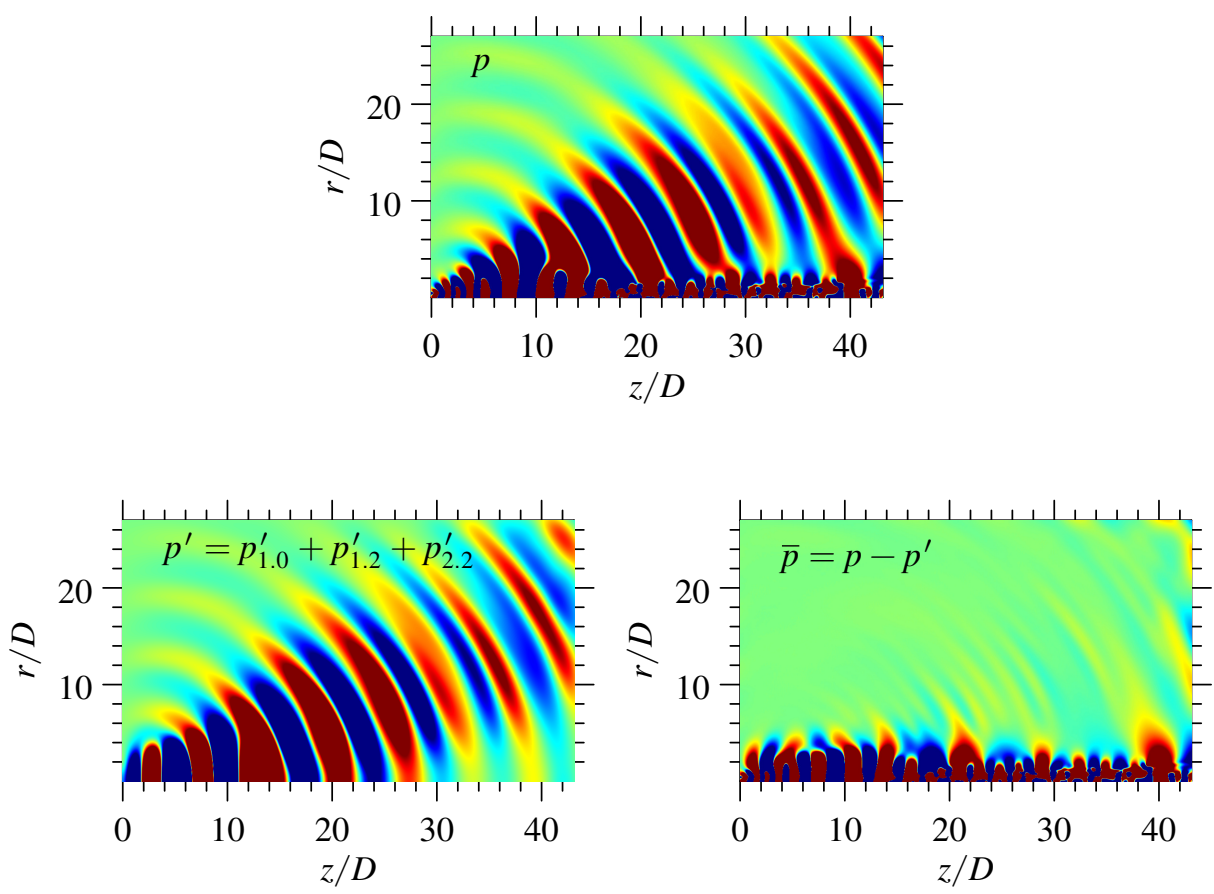


\section{NRBF sources}

Axial NRBF source $f_{z}$ at $\omega=1.2$

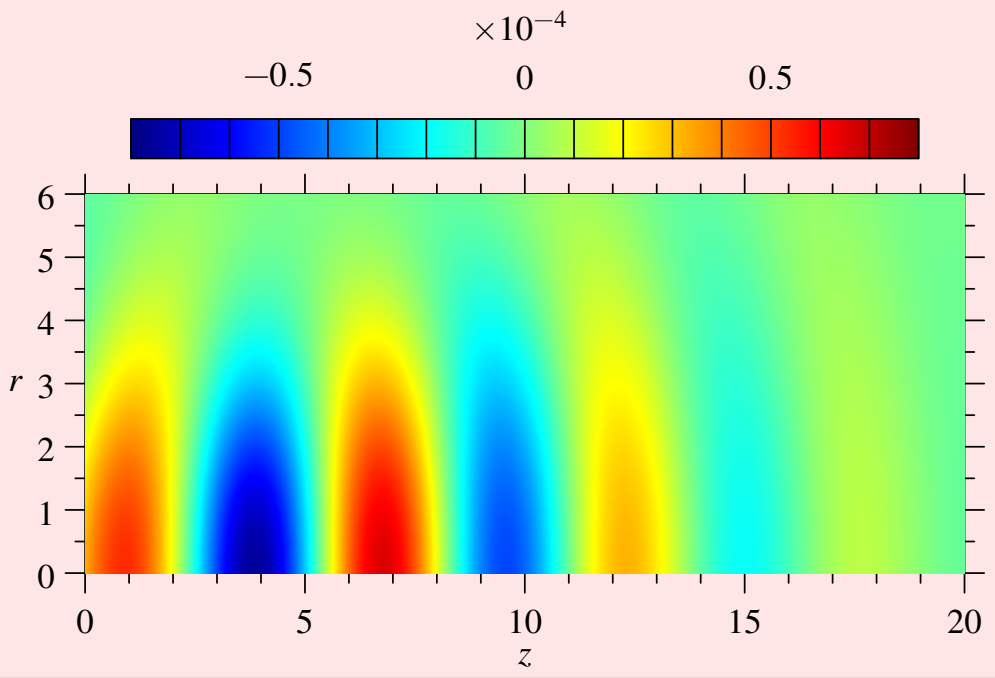




\section{NRBF sources}

Radial NRBF source $f_{r}$ at $\omega=1.2$

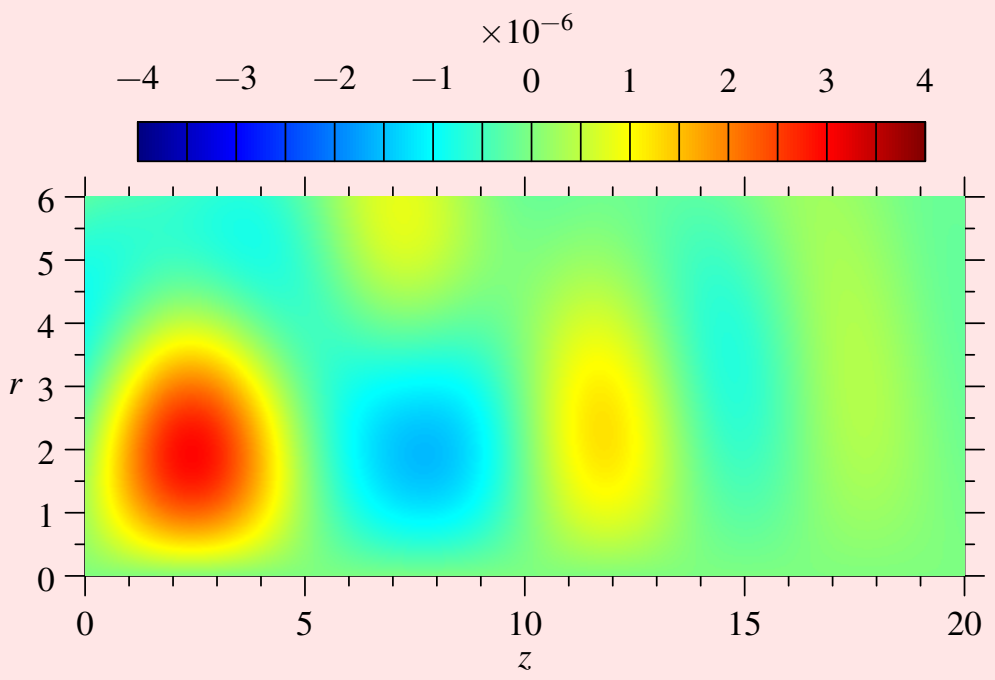




\section{Source validation}

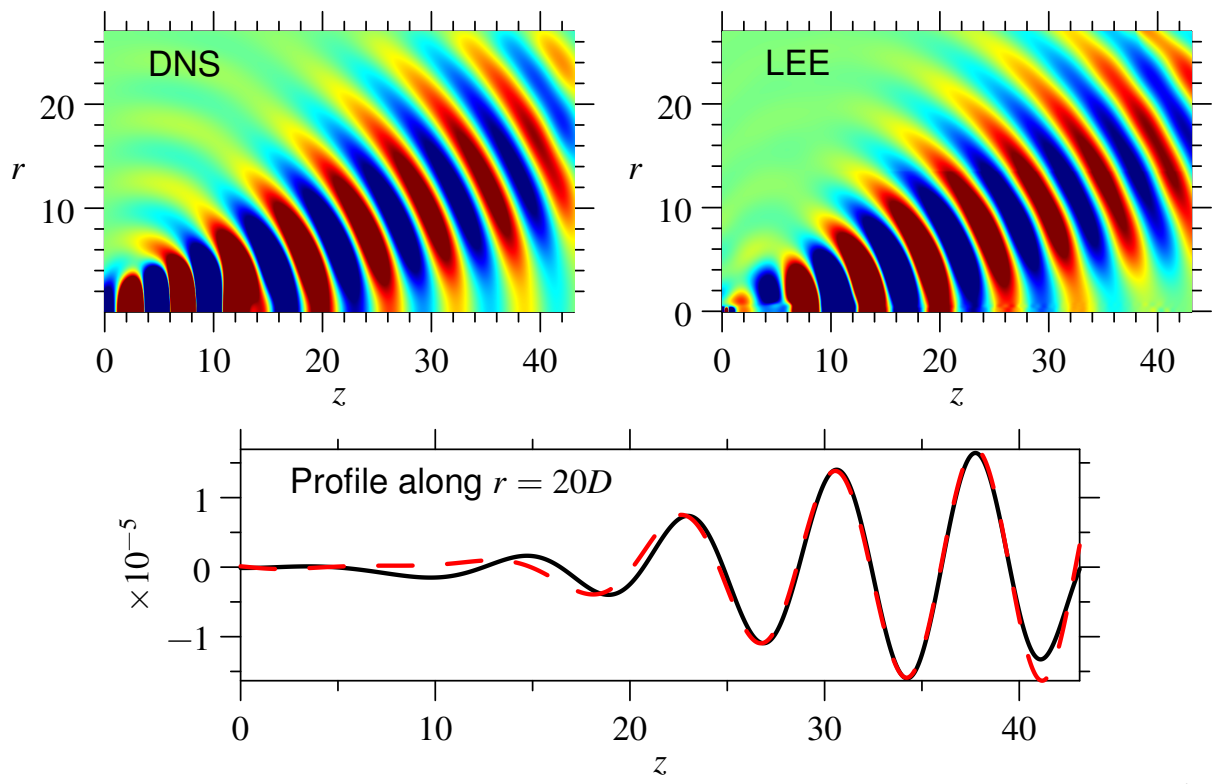




\section{Source identification: shear-noise}

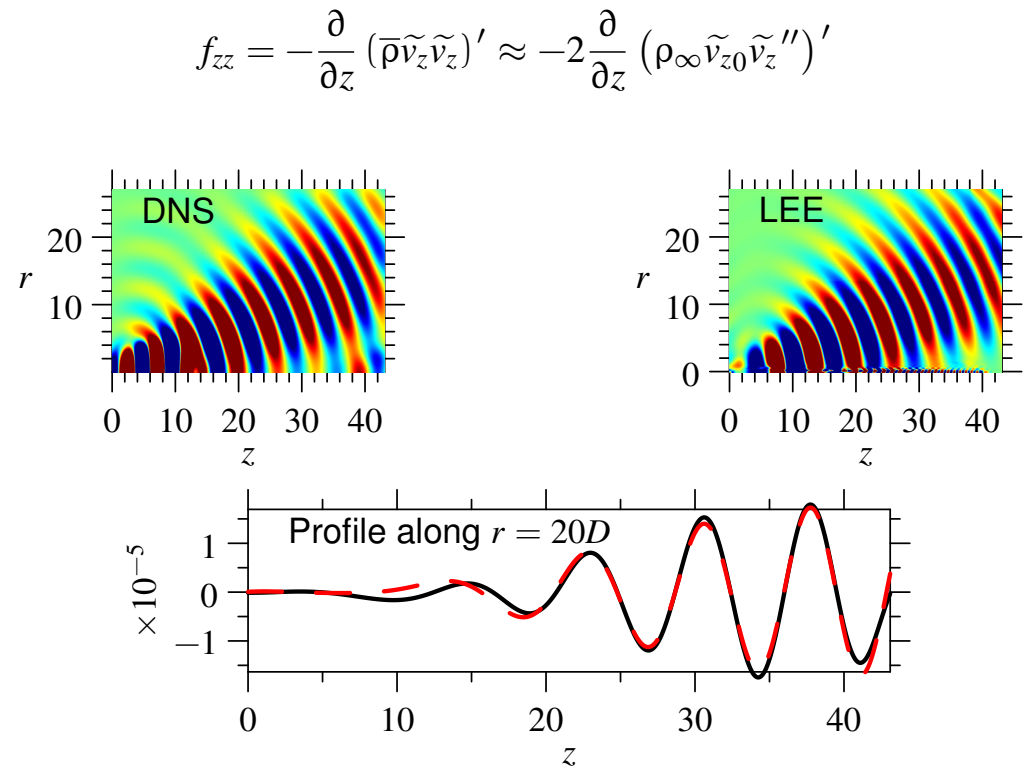




\section{Part III}

\section{Sound sources in a turbulent jet}




\section{Flow analysis}

Full density field

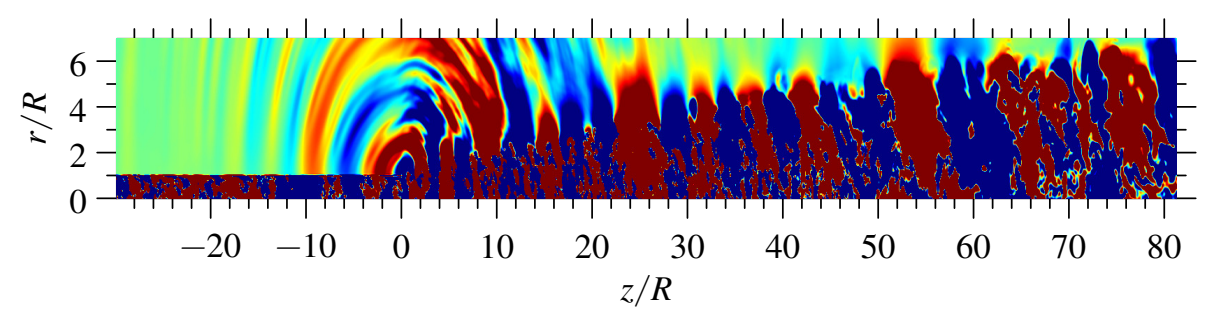




\section{Flow analysis}

Full density field

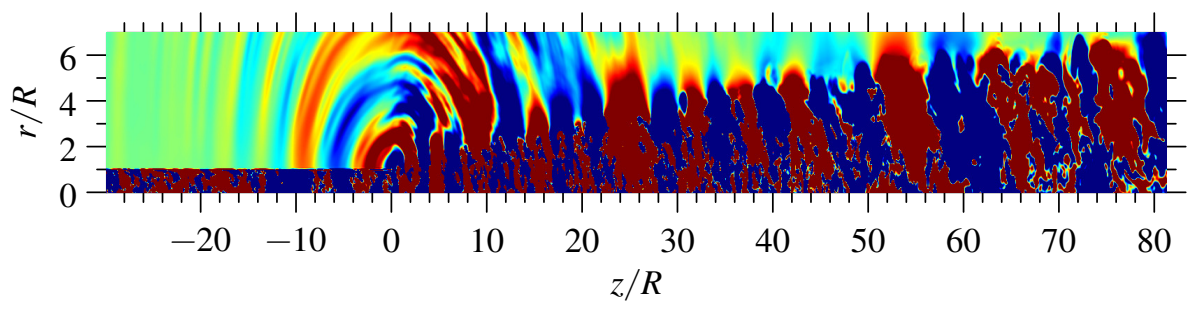

Density field, modes 0 and $1,\left|S t_{D}\right|<4$

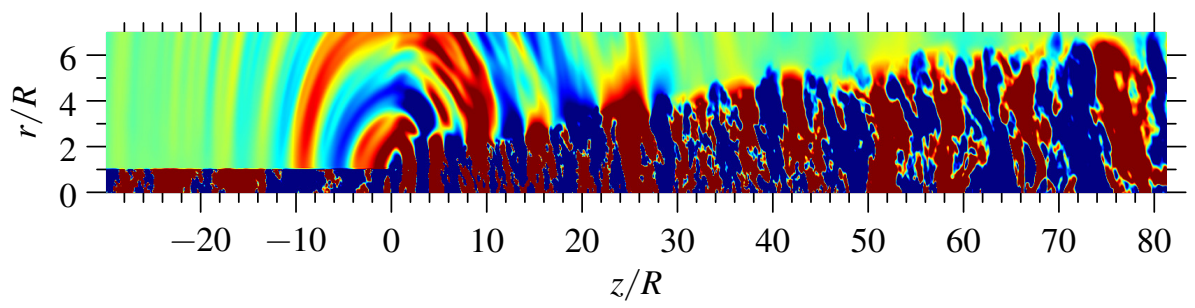




\section{Flow decomposition: $n=0, S t_{D}=0.5$}
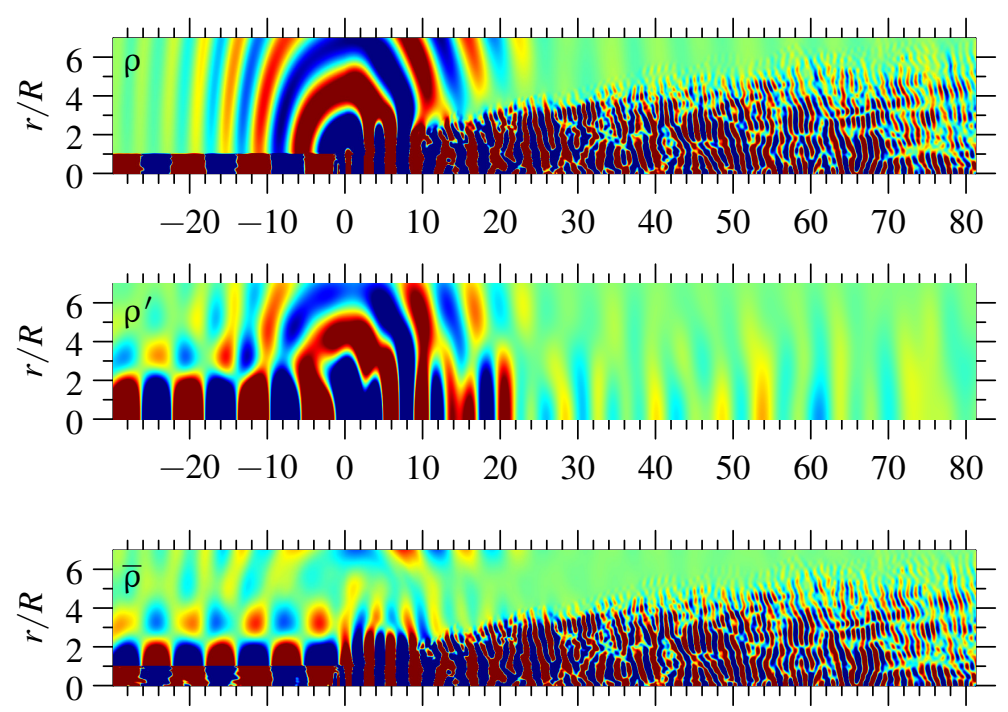

$\begin{array}{lllllllllll}-20 & -10 & 0 & 10 & 20 & 30 & 40 & 50 & 60 & 70 & 80\end{array}$ 


\section{Flow decomposition: $n=0, S t_{D}=0.8$}
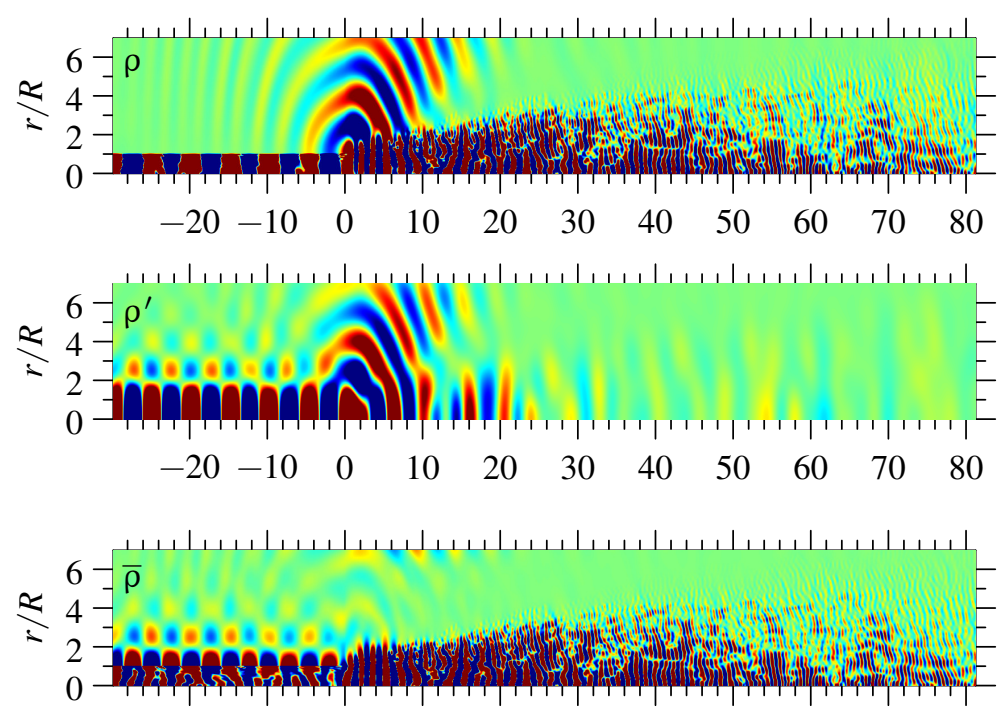

$\begin{array}{lllllllllll}-20 & -10 & 0 & 10 & 20 & 30 & 40 & 50 & 60 & 70 & 80\end{array}$ 


\section{Flow decomposition: $n=0, S t_{D}=1.45$}
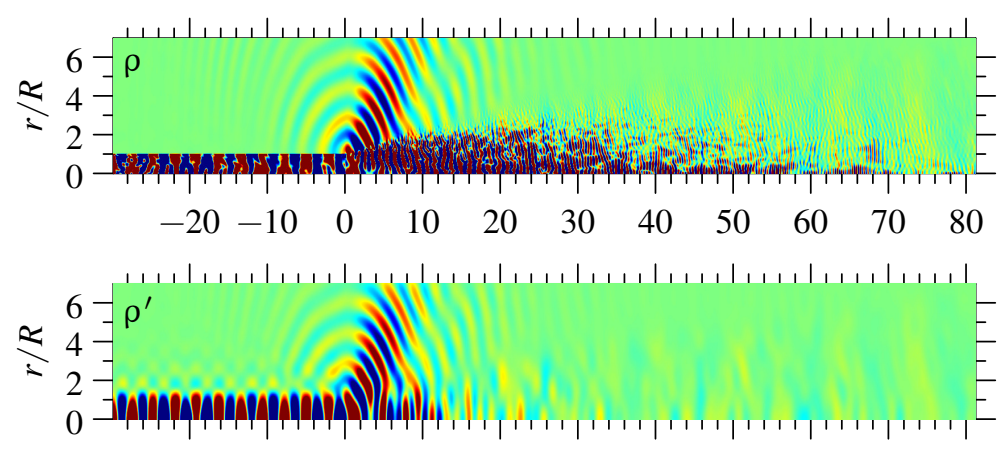

$\begin{array}{lllllllllll}-20 & -10 & 0 & 10 & 20 & 30 & 40 & 50 & 60 & 70 & 80\end{array}$

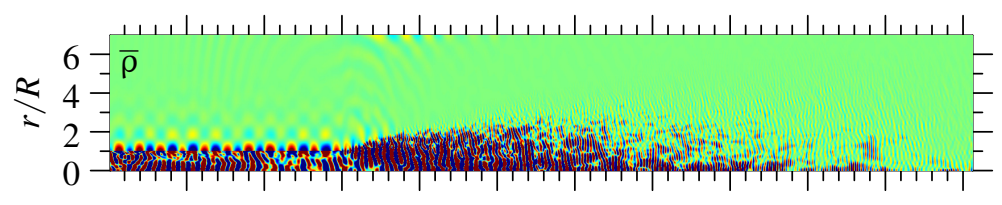

$\begin{array}{lllllllllll}-20 & -10 & 0 & 10 & 20 & 30 & 40 & 50 & 60 & 70 & 80\end{array}$ 
Flow decomposition: $n=0,0.5 \leqslant\left|S t_{D}\right|<4.0$
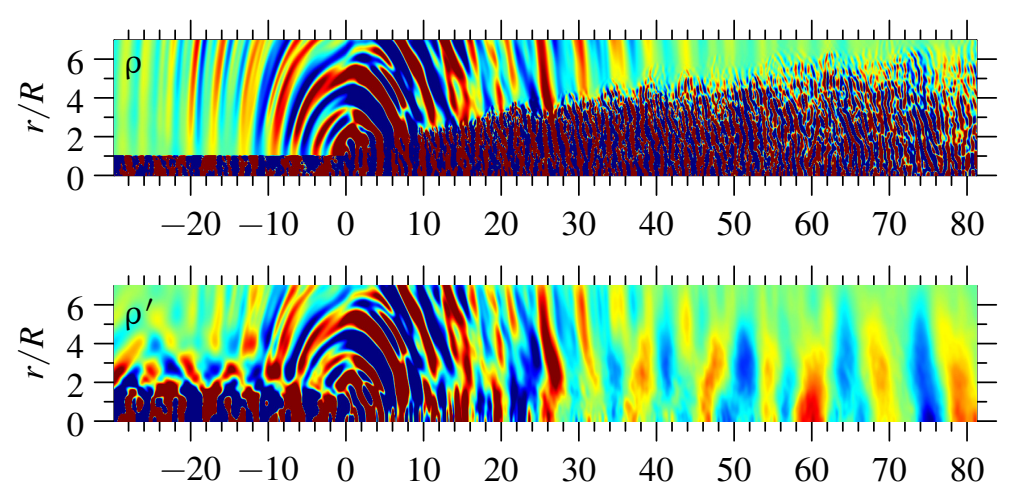

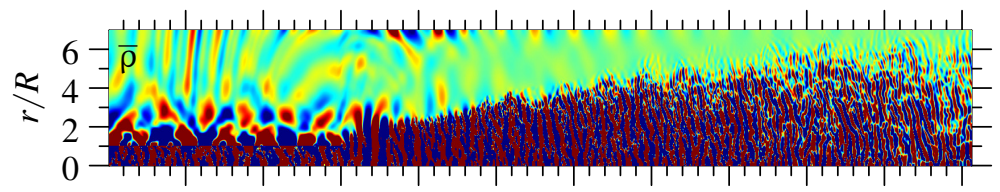

$\begin{array}{lllllllllll}-20 & -10 & 0 & 10 & 20 & 30 & 40 & 50 & 60 & 70 & 80\end{array}$ 
Flow decomposition: $n=1,0.5 \leqslant\left|S t_{D}\right|<4.0$
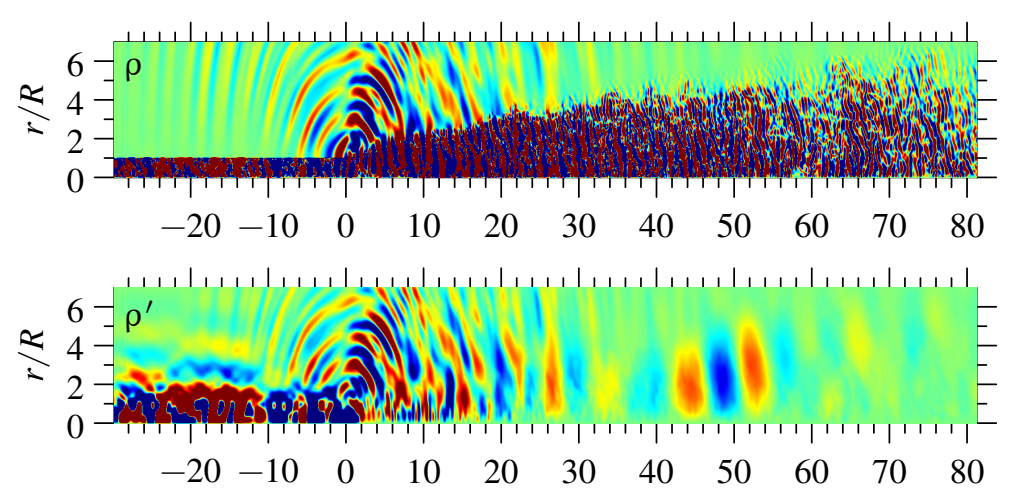

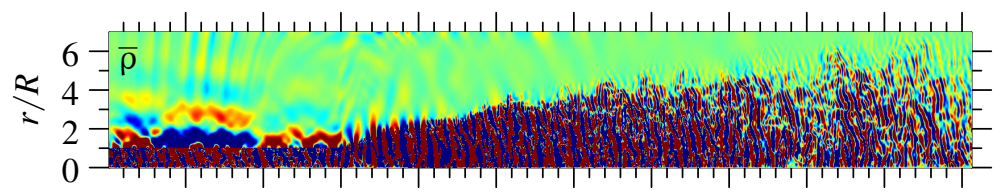

$\begin{array}{lllllllllll}-20 & -10 & 0 & 10 & 20 & 30 & 40 & 50 & 60 & 70 & 80\end{array}$ 


\section{NRBF sources: $S t_{D}=0.5$}

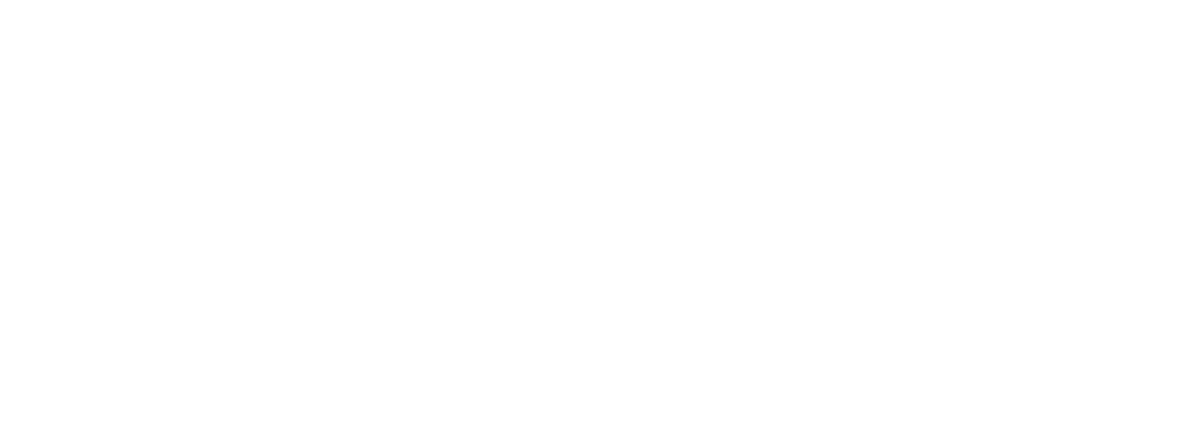


NRBF sources: $S t_{D}=1.1$

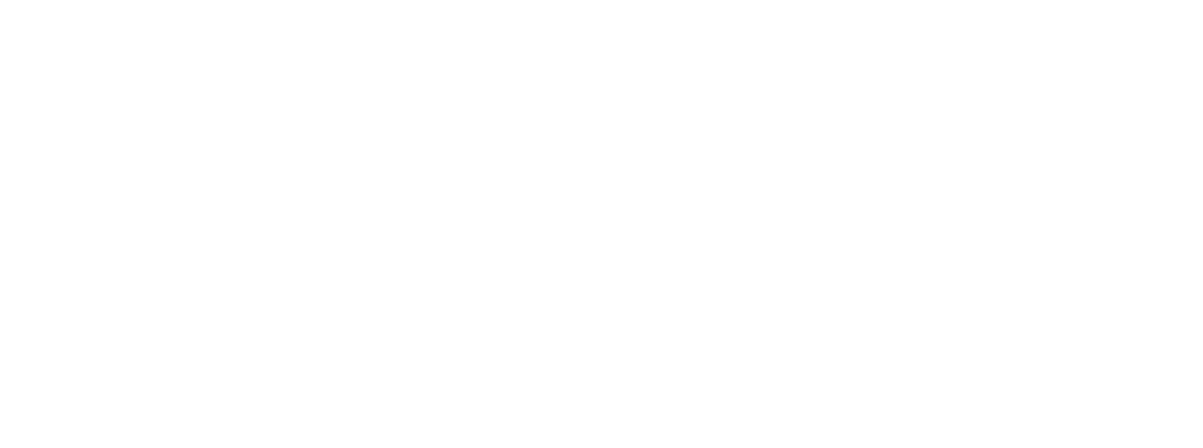




\section{NRBF sources: $S t_{D}=1.45$}

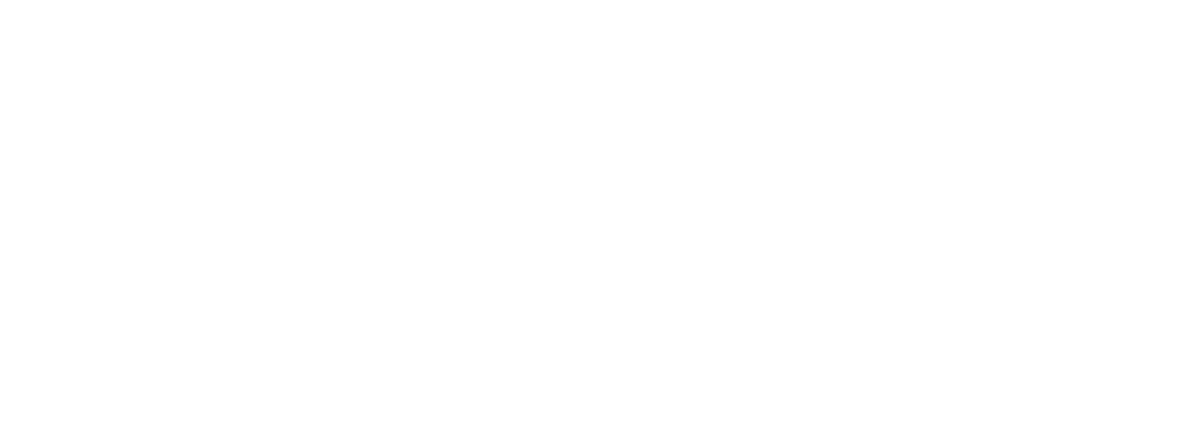




\section{NRBF sources: $S t_{D}=2.55$}

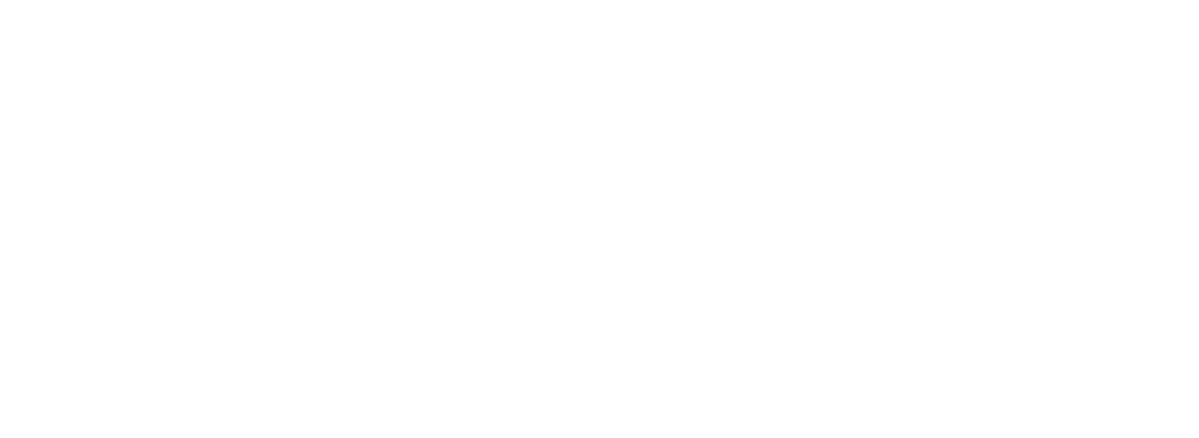


Qualitative validation: $n=0, S t_{D}=1.1$
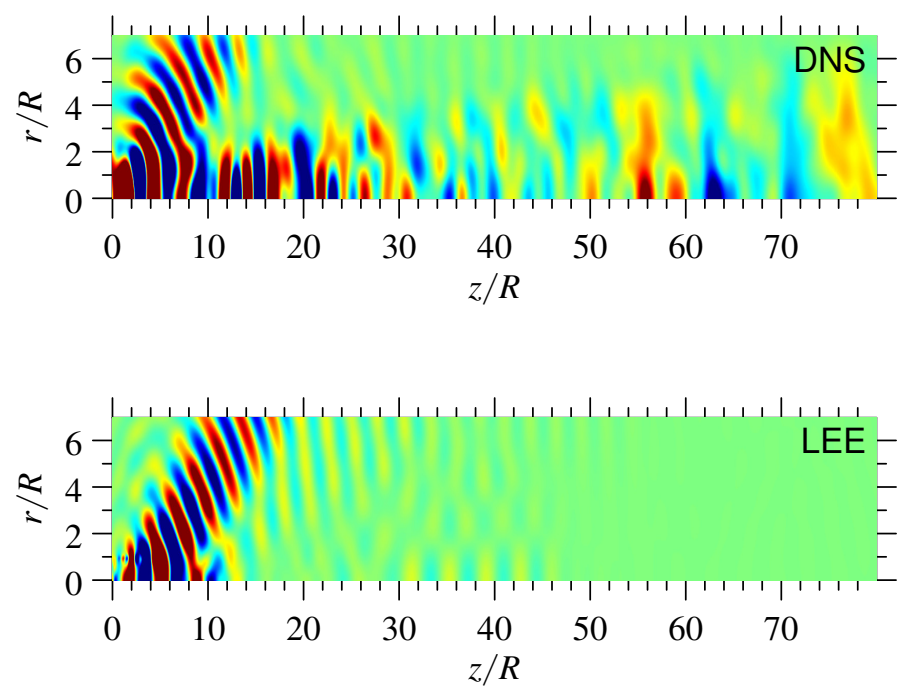


\section{Conclusion}

- Algorithm to compute the NRBF sources at multiple frequencies

(2) In laminar jet: linear shear noise term is major source

(3) NRBF decomposition and sources for a fully turbulent jet

- wavepackets

- monopole

- vortex pairing 


\title{
Acknowledgements
}

\author{
EPSRC \\ Engineering and Physical Sciences \\ Research Council
}

Thank you! 